See Article page 2180.

\section{Commentary: If a little is good, more must be better?}

\author{
Vincent Olshove, CCP, and Bahaaldin Alsoufi, MD
}

In the current issue of the Journal, Liu and colleagues ${ }^{1}$ explore optimal perfusate oxygenation $(\mathrm{PpO} 2)$ in children who underwent cardiac surgery and evaluated the relationship among $\mathrm{PpO} 2$, systemic inflammatory response syndrome, and geographical altitude. They defined high altitude as greater than $500 \mathrm{~m}$ above sea level, which is lower than the usual definition of high altitude at approximately $2400 \mathrm{~m}$ above sea level. In this study, they showed a steeper association between $\mathrm{PpO}_{2}$ and the risk of severe systemic inflammatory response syndrome in patients from a low altitude than in patients from a high altitude. Their analysis suggested that patients from a low altitude might have the best outcomes with lower doses of $\mathrm{PpO} 2$, whereas patients from a high altitude might have the best outcomes with the optimal $\mathrm{PpO} 2$ of approximately 250 to $350 \mathrm{~mm} \mathrm{Hg}{ }^{1}$

It is not uncommon for one to approach a problem from the perspective that if a little is good, more must be better. However, Liu and colleagues ${ }^{1}$ and others ${ }^{2,3}$ have shown us that this is not necessarily true when it comes to cardiopulmonary bypass (CPB) in congenital heart disease. In a study by Caputo and colleagues, ${ }^{4} 67$ cyanotic patients were randomized to receive controlled normoxic (50$80 \mathrm{~mm} \mathrm{Hg}$ ) or hyperoxic $(150-180 \mathrm{~mm} \mathrm{Hg}) \mathrm{CPB}$. Termination of CPB was 100 to $110 \mathrm{~mm} \mathrm{Hg}$ for the normoxic group and the hyperoxic group with fraction of inspired oxygen $\left(\mathrm{FiO}_{2}\right)$ on $\mathrm{CPB}$ up to $100 \%$ to maintain saturations greater than $95 \%$. Further, anesthesia was induced at an $\mathrm{FiO}_{2}$ of 0.21 in the normoxic group and 0.50 in the hyperoxic group.

\footnotetext{
From the Department of Cardiovascular and Thoracic Surgery, University of Louisville School of Medicine, Norton Children's Hospital, Louisville, Ky.

Disclosures: The authors reported no conflicts of interest.

The Journal policy requires editors and reviewers to disclose conflicts of interest and to decline handling or reviewing manuscripts for which they may have a conflict of interest. The editors and reviewers of this article have no conflicts of interest.

Received for publication July 18, 2020; revisions received July 18, 2020; accepted for publication July 20, 2020; available ahead of print July 23, 2020.

Address for reprints: Bahaaldin Alsoufi, MD, Department of Cardiovascular and Thoracic Surgery, University of Louisville School of Medicine, Norton Children's Hospital, 201 Abraham Flexner Way, Suite 1200, Louisville, KY 40202 (E-mail: balsoufi@hotmail.com).

J Thorac Cardiovasc Surg 2021;161:2191-2

$0022-5223 / \$ 36.00$

Copyright (C) 2020 by The American Association for Thoracic Surgery

https://doi.org/10.1016/j.jtcvs.2020.07.060
}

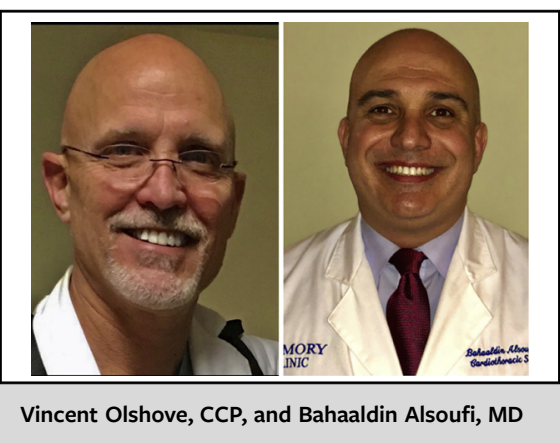

$$
\begin{aligned}
& \text { CENTRAL MESSAGE } \\
& \text { Multiple variables including hy- } \\
& \text { peroxygenation affect systemic } \\
& \text { inflammatory response syn- } \\
& \text { drome with CPB. Electronic } \\
& \text { perfusion records and perfusion } \\
& \text { registries might provide a way to } \\
& \text { compare perfusion strategies } \\
& \text { and define best practices. }
\end{aligned}
$$

They showed that controlled reoxygenation on starting CPB was associated with reduced myocardial damage, oxidative stress, and cerebral and hepatic injury compared with hyperoxic bypass. In their study, there was no discussion of actual peak $\mathrm{PpO}_{2}$. A study by $\mathrm{Babu}$ and colleagues 5 randomized 31 cyanotic children to 1 of 2 groups: Group 1 had CPB initiation with $\mathrm{FiO}_{2} 0.21$, and after 1 minute of full bypass, $\mathrm{FiO}_{2}$ was increased at increments of 0.1 per minute until reaching 0.6. Group 2 was initiated using $\mathrm{FiO}_{2}$ 0.6. After 5 minutes in both groups, arterial blood gas analysis was performed and $\mathrm{PpO}_{2}$ was maintained at 200 to $300 \mathrm{~mm} \mathrm{Hg}$. They found that a controlled oxygenation protocol was associated with significantly lower postoperative creatine phosphokinase myocardial band (CPK-MB) levels and decreased ventilation time. Again, they did not discuss peak $\mathrm{PpO}_{2}$ in their study. ${ }^{5}$

There are numerous factors that appear to influence systemic inflammation reactions after $\mathrm{CPB}$, and these include contact of blood with the CPB circuit, surgical operation, blood loss, and blood/blood product administration. Ultimately, a team approach toward congenital heart surgery to confront these issues should be applied. The problem with CPB is there is no accepted definition of optimal perfusion, and there is a continuum of quality ranging from adequate, sufficient, or minimally acceptable that progresses through superior, optimal, or maximal. ${ }^{6}$ Minimizing 
systemic inflammatory response is the goal for every CPB case. ${ }^{7}$ Ultimately, the approach is a "prescriptive perfusion" individualized to each patient. Circuit miniaturization, including biocompatible oxygenator (integrated filter), and tubing (arterial, venous, sucker, and sumps) can lessen the overall surface area of the bypass circuit, minimizing blood exposure and reducing the quantity administered while maximizing the desired target hematocrit on CPB. Ultrafiltration, especially when blood products are used in the prime, should be considered and the prime composition normalized to mimic patient arterial blood gas and electrolyte analysis and maintained for initiation of CPB. Bypass should be initiated with a $\mathrm{PpO} 2$ as close to the patient's partial pressure of oxygen and maintained within a relatively normoxic target, as long as adequate oxygen delivery is achieved. Best efforts should be given toward reduction in blood transfusions through meticulous hemostasis, circuit miniaturization, blood sampling (volume and quantity), and establishment of and adherence to guidelines for blood and platelet transfusions.

The one issue in the study by Liu and colleagues ${ }^{1}$ is that it was based on the highest $\mathrm{PpO}_{2}$ during aortic occlusion, and that one time point after aortic occlusion may be too late for cause and effect.

One concern with the work looking at normoxic and hyperoxic CPB research is the variation in the definition of each. Further, because many aspects of the perfusion technique can contribute to the overall inflammatory response, there should be greater detail provided in the perfusion technique, especially to prime volume, circuitry used, and blood products used, in addition to the $\mathrm{PpO}_{2}$ and how that component was managed. With the advent of electronic perfusion records, and the use of inline blood gas analyzers, the ability to capture actual $\mathrm{PpO}_{2}$ at 10 seconds or less time intervals, and at precise time points, is now available. The significance of being able to do clinical research and report average $\mathrm{PpO} 2$ before crossclamp, average during crossclamp, average or actual at crossclamp removal, and at termination of CPB should lend a more significant evaluation of techniques and help define best practice in the future. The development of a pediatric/congenital perfusion registry to submit perfusion-related parameters is necessary to begin to define best practices so that we may eventually be able to compare practices and better define optimal perfusion.

Meanwhile, until we are able to overcome these challenges and variations in CPB research to produce tangible evidence of best perfusion practice, the studies by Liu and colleagues ${ }^{1}$ and others suggest that instead of "if a little is good, more must be better," we should consider that possibly "less is more."

\section{References}

1. Liu H, Hu Y, Zheng S, Chen T, Zeng Z, Wu D, et al. Effect of perfusate oxygenation on inflammatory response in congenital heart disease children from low versus high altitude. J Thorac Cardiovasc Surg. 2021;161:2180-90.

2. Morita K. Surgical reoxygenation injury of the myocardium in cyanotic patients: clinical relevance and therapeutic strategies by normoxic management during cardiopulmonary bypass. Gen Thorac Cardiovasc Surg. 2012;60:549-56.

3. Kagawa H, Morita K, Uno Y, Ko Y, Matsumura M, Kinouchi K, et al. Inflammatory response to hyperoxemic and normoxemic cardiopulmonary bypass in acyanotic pediatric patients. World J Pediatr Congenit Heart Surg. 2014;5: $541-5$.

4. Caputo M, Mokhtari A, Rogers C, Panayioto N, Chen Q, Ghorbel M, et al. The effects of normoxic versus hyperoxic cardiopulmonary bypass on oxidative stress and inflammatory response in cyanotic pediatric patients undergoing open cardiac surgery: a randomized controlled trial. J Thorac Cardiovasc Surg. 2009;138: 206-14.

5. Babu B, Bhat S, Prabuswamy H, Kamalapurkar G, Libu GK, Shilpa S, et al. Controlling oxygenation during initiation of cardiopulmonary bypass: can it improve immediate postoperative outcomes in cyanotic children undergoing cardiac surgery? A prospective randomized study. World J Pediatr Congenit Heart Surg. 2012;3:310-6.

6. Murphy G, Hessel E, Groom R. Optimal perfusion during cardiopulmonary bypass: an evidence-based approach. Anesth Analg. 2009;13:113-7.

7. Durandy Y. Minimizing systemic inflammation during cardiopulmonary bypass in the pediatric population. ASAIO J. 2014;38:11-8. 